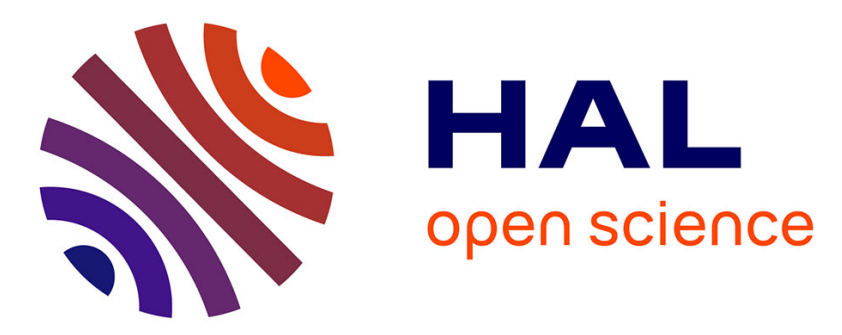

\title{
Detection of Hydrogen in Neutron Irradiated Nickel using Positron Lifetime Spectroscopy
}

Troyo Dimov Troev, Toshimasa Yoshiie, Chunqing He, Koichi Sato, Qiu Xu, Stela Georgieva Peneva

\section{To cite this version:}

Troyo Dimov Troev, Toshimasa Yoshiie, Chunqing He, Koichi Sato, Qiu Xu, et al.. Detection of Hydrogen in Neutron Irradiated Nickel using Positron Lifetime Spectroscopy. Philosophical Magazine, 2009, 89 (14), pp.1183-1195. 10.1080/14786430902915404 . hal-00514018

\section{HAL Id: hal-00514018 \\ https://hal.science/hal-00514018}

Submitted on 1 Sep 2010

HAL is a multi-disciplinary open access archive for the deposit and dissemination of scientific research documents, whether they are published or not. The documents may come from teaching and research institutions in France or abroad, or from public or private research centers.
L'archive ouverte pluridisciplinaire HAL, est destinée au dépôt et à la diffusion de documents scientifiques de niveau recherche, publiés ou non, émanant des établissements d'enseignement et de recherche français ou étrangers, des laboratoires publics ou privés. 


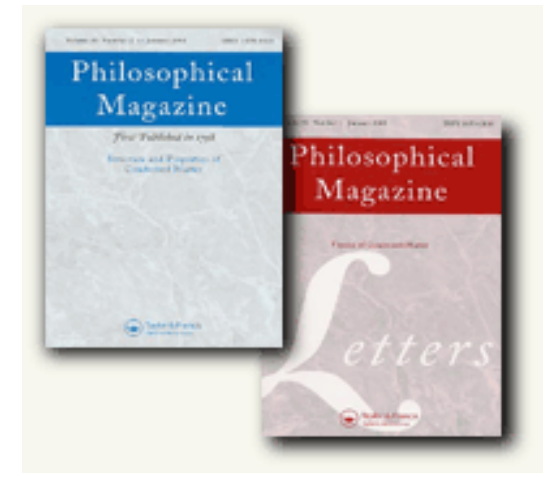

\section{Detection of Hydrogen in Neutron Irradiated Nickel using Positron Lifetime Spectroscopy}

\begin{tabular}{|c|c|}
\hline Journal: & Philosophical Magazine \& Philosophical Magazine Letters \\
\hline Manuscript ID: & TPHM-08-Nov-0443.R1 \\
\hline Journal Selection: & Philosophical Magazine \\
\hline $\begin{array}{l}\text { Date Submitted by the } \\
\text { Author: }\end{array}$ & 02-Mar-2009 \\
\hline Complete List of Authors: & $\begin{array}{l}\text { TROEV, TROYO; Bulgarian Academy of Sciences, Institutte for } \\
\text { Nuclear Research and Nuclear Energy } \\
\text { Yoshiie, Toshimasa; Research Reactor Institute Kyoto University, } \\
\text { Material Sciences } \\
\text { He, Chunqing; Research Reactor Institute Kyoto University, Material } \\
\text { Sciences, Material Sciences; Research Reactor Institute Kyoto } \\
\text { University, Material Sciences } \\
\text { Sato, Koichi; Kyoto University, Research Reactor Institute; Kyoto } \\
\text { University, Research Reactor Institute } \\
\text { Xu, Qiu; Kyoto University, Research Reactor Institute } \\
\text { Peneva, Stela; Bulgarian Academy of Sciences,, Institutte for } \\
\text { Nuclear Research and Nuclear Energy }\end{array}$ \\
\hline Keywords: & $\begin{array}{l}\text { irradiation effects, neutron irradiation, nickel, positron annihilation, } \\
\text { radiation effects }\end{array}$ \\
\hline Keywords (user supplied): & irradiation effects, neutron irradiation, nickel \\
\hline
\end{tabular}

\section{s ScholarONE" \\ Manuscript Central}




\title{
Detection of Hydrogen in Neutron Irradiated Nickel using Positron Lifetime Spectroscopy
}

\author{
C. $\mathrm{He}^{1}$, T. Yoshiie ${ }^{1}$, Q. Xu ${ }^{1}$, K. Sato ${ }^{1}$, S. Peneva ${ }^{2}$ and T. D. Troev ${ }^{2 *}$ \\ ${ }^{1}$ Research Reactor Institute, Kyoto University, \\ Kumatori-Cho, Sennan-Gun, Osaka-Fu 590-0494, Japan \\ ${ }^{2}$ Institute for Nuclear Research and Nuclear Energy, \\ Bulgarian Academy of Sciences, 72 Tzarigradsko, Chaussee, Sofia 1784, Bulgaria
}

\begin{abstract}
Hydrogen in nano-voids in neutron-irradiated nickel has been detected using positron annihilation lifetime spectroscopy (PALS). As positron lifetime is greatly affected by nano-voids bound with hydrogen, special attention was paid to the analysis. The positron lifetime of neutron-irradiated nickel at higher irradiation dose increased with the dose, which is an indicator for vacancy clusters (nano-voids) formation in the lattice. The introduction of hydrogen in wellannealed nickel by electrical charging resulted also in an increase in positron lifetime due to vacancy formation. In neutron-irradiated nickel specimens, hydrogen charging shortened the positron lifetime from 456 to 334 ps (irradiation dose: $3 \times 10^{-3}$ dpa). Isochronal annealing behavior of hydrogen-charged nickel and neutron-irradiated nickel has also been studied. Positron trapping rate was calculated using a simple trapping model. Thermal desorption spectroscopy [TDS] has been used for investigation of hydrogen behavior in non-irradiated hydrogen charged nickel.
\end{abstract}

PACS: 78.70.Bj; 61.80.Bg; 61.80.Hg; 61.72.Cc

Keywords: positron annihilation, hydrogen charging, neutron irradiation, vacancy-cluster, nickel

* Corresponding author: tel. +3592 97401 42; fax: +3592975 3619.

e-mail address: troev @ inrne.bas.bg 


\section{Introduction}

The PALS method due to the high positron trapping probability is sensitive to defect concentration as low as $10^{-6}$ [1]. The advances in PALS have emphasized the usefulness of the positron method in defect spectroscopy studies, as a source of both qualitative and quantitative information about defects on an atomic scale. At present the PALS is a well-known method for studying atomic defects, such as vacancies, vacancy-clusters (nano-voids), dislocations, precipitates as well as defects containing gas atoms and has proved to give valuable information on electronic and ionic structures. In a perfect crystal, positrons are delocalized and annihilated via a Bloch state with electrons in the matrix. In condensed materials containing vacancies or nano-voids, the electron density is much lower than that in the matrix. The lifetime of positrons correlates with the magnitude of the electron density at the site of positron annihilation and give information for the open volumes at the vacancy defects. Positron trapping depends on the rearrangement of ions and conduction electrons at the defects as well as the reduction of positron kinetic energy and variation of the electron-positron correlation energy. The positrons are preferentially trapped by lattice defects, due to the strong Coulomb repulsion from the ion cores and are annihilated (with electrons) near the defects [2, 3]. Positron annihilation characteristics, such as positron lifetimes, intensities, and Doppler broadening line shape parameters, depend on the defect type and the chemical environment of the defects [4-7]. In the case of nanovoids, positron lifetime increases with nanovoid size. Hydrogen is produced in fission and fusion reactor materials, respectively. In a spallation neutron source, the target materials also contain hydrogen as a result of the irradiation by protons. That is why it is important to understand the behavior of hydrogen atoms in these materials $[3,8,9]$. Recently it has been demonstrated that the coincidence Doppler broadening $[\mathrm{CDB}]$ of positron annihilation radiation is effective for detection of hydrogen atoms in ion irradiated nickel nano-voids (10). Hydrogen is produced in nuclear materials during neutron irradiation via nuclear reaction $(n, p)$. In the present study, we 
are interested in hydrogen charging effects in well annealed unirradiated and in neutronirradiated nickel studied by PALS as well as in model calculations of positron states in nickel nano-voids containing hydrogen. To our knowledge, the number of hydrogen atoms in nanovoids was experimentally estimated for the first time. If the vacancies and nanovoids contain gas atoms, the positron lifetime decreases due to the overlap of electrons of gas atoms with trapped positrons $[10,11]$. However, the detection of hydrogen in metals by PALS has not been quantitatively analyzed except by Ohkubo et al. [11]. In the present paper in addition to the PALS the method of thermal desorbtion spectroscopy was performed for detection of the hydrogen desorption in non-irradiated hydrogen charged nickel. The main purpose of this paper is to provide information for detection of hydrogen in neutron-irradiated nickel. In particular, we shall discuss the positron interaction in hydrogen charged neutron irradiated nickel. The rest of this paper is organized as follows: The experimental procedure is described in Secs. 2. Experimental results are presented and compared with theoretical ones in Sec. 3. The physical trend observed in the experiments is discussed based of neutron irradiated nickel as well as the hydrogen charging and isochronal annealing of unirradiated and irradiated nickel samples in Secs. 3.1 to 3.4 ; Section 4 gives a short summary.

\section{Experimental procedure}

High purity nickel specimens $(99.99 \%$, Johnson \& Matthey) were cold-rolled to $0.1 \mathrm{~mm}$ thickness. Circular specimens with a diameter of 3 or $5 \mathrm{~mm}$ were punched out of these nickel sheets. The specimens were annealed for $1 \mathrm{~h}$ at $1173 \mathrm{~K}$ in a vacuum of about $133.3 \times 10^{-6} \mathrm{~Pa}$. The average grain size after annealing was about $0.03 \mathrm{~mm}$. Nickel specimens were irradiated at 573 $\mathrm{K}$ with fission neutrons at the Materials Controlled Irradiation Facility of the Kyoto University Reactor (KUR) [12] to a dose of $6.7 \times 10^{-6}, 4.0 \times 10^{-5}, 1.0 \times 10^{-4}$ or $3.0 \times 10^{-3} \mathrm{dpa}$ (displacement per 
atom; threshold energy: $24 \mathrm{eV}$ ). Under these irradiation conditions, the generation of gas atoms by nuclear reactions was negligibly small.

The electrochemical hydrogen charging of well-annealed nickel and neutron-irradiated nickel was conducted in a bath of $4 \mathrm{~N} \mathrm{H}_{2} \mathrm{SO}_{4}$ under a current density of about $20 \mathrm{~mA} / \mathrm{cm}^{2}$. An isochronal annealing for 1-hour was performed on the specimens in a vacuum after hydrogen charging. Positron annihilation lifetime was measured at room temperature using a fast-fast coincidence system, whose lifetime resolution (FWHM) was 190 ps. The counting rate was about 80 cps. The lifetime spectra were collected with a total count between 1 and $3 \times 10^{6}$. Several spectra were accumulated in order to ensure the reproducibility of the data. The positron lifetime spectra were analyzed using the programs Resolution and Positronfit [13].

Thermal desorption spectrum (TDS) of hydrogen-charged nickel was taken with a thermal desorption spectroscope (EMD-WA1000S/W, ESCO Ltd.) from $333 \mathrm{~K}$ to $783 \mathrm{~K}$ at a rate of 1 K/s under a vacuum of $399.9 \times 10^{-9} \mathrm{~Pa}$.

\section{Results and discussion}

\subsection{Neutron-irradiated nickel}

The lifetime of a trapped positron in nickel defects is larger than that of the positron being annihilated in the free state, because the density of electrons inside a vacancy is less than that the interstitial region. We established that the lifetime of a trapped positron is sensitive to the size of vacancy defects as well as to the number of hydrogen atoms in nickel defects.

As shown in Table 1, the positron lifetime spectrum for unirradiated nickel includes a single lifetime component of $110 \mathrm{ps}$, which agrees well with the calculated lifetime of $110 \mathrm{ps}$ for a perfect nickel lattice and the results of Kuramoto et al. [14]. In nickel neutron-irradiated to a dose of $6.7 \times 10^{-6} \mathrm{dpa}$, the positron lifetime spectra were decomposed into two components, $\tau_{1}$ and $\tau_{2}$. The long positron lifetime $\tau_{2}$ corresponds to the annihilation of trapped positrons in vacancies 


\subsection{Hydrogen charging and isochronal annealing of unirradiated nickel}

In the papers $[11,18,19]$ it has been reported that hydrogen charging could induce a large range of defects, such as mono-vacancy, vacancy-clusters and dislocations in the lattice of metals and alloys. The positron lifetime experimental results from detection of the effects of hydrogen charging on an unirradiated nickel specimen are shown in Fig. 1. 
Fig. 1. Positron lifetime and its intensity as a function of the electrical hydrogen charging time in nickel

The long lifetime spectra have been resolved into two components. The positron lifetime increases with hydrogen charging time, while its intensity decreases slightly. The lifetime, $\tau_{2}$, is saturated after $9 \mathrm{~h}$ of hydrogen charging and maintains a constant value of about 164 ps. In Fig. 2 is shown the TDS of hydrogen as a function of temperature obtained after $10 \mathrm{~h}$ of electrochemical hydrogen charging. Our results show that the main hydrogen desorption peak is at $423 \mathrm{~K}$.

Fig. 2. Thermal desorption spectrum for electrically hydrogen-charged nickel with a linear temperature ramp of $1 \mathrm{~K} / \mathrm{s}$.

The hydrogen detrapping energy in the hydrogen-charged nickel specimen determined from TDS data is equal to $0.91 \mathrm{eV}$. This value is comparable to the detrapping energy of $0.98 \mathrm{eV}$ for mono-vacancy bound with one hydrogen atom in nickel [20-22]. It is interesting to note that recent theoretical calculations based on the atom superposition method [11] and the simulations [23] show that the positron lifetime of a nickel mono-vacancy containing one hydrogen was 163 [11] and $158 \mathrm{ps,}$, respectively. We conclude that the long positron lifetime of $164 \mathrm{ps}$ is attributable to the positron annihilation in nickel vacancy bound with one hydrogen atom.

The results from investigation of the annealing behavior of the hydrogen-charged nickel by PALS are shown in Fig. 3. It is seen that the long lifetime increases from 164 to 223 ps, while its intensity decreases from $40 \%$ to $23 \%$, upon annealing at $373 \mathrm{~K}$ in vacuum for $1 \mathrm{~h}$. Our results indicate for the first time that nano-voids containing of 3-5 vacancies have been formed by the migration of vacancies, induced by the release of hydrogen upon annealing at $373 \mathrm{~K}$. 
Fig. 3. Effect of isochronal annealing on the positron lifetime and intensity in hydrogen-charged nickel.

This contradicts the previous results [24], which indicate that the activation energy for migration of vacancies in nickel (purity: 99.99\%, as in our specimens) was $1.25 \mathrm{eV}$, which allows vacancy clustering above $473 \mathrm{~K}$. The vacancies in ultrahigh-purity nickel are mobile at $300 \mathrm{~K}$, so the clustering of vacancies took place around room temperature [11]. Our results could be explained in the following way: Hydrogen charging induces a high density of vacancies containing hydrogen at specific sites, such as near grain boundaries and the surface, since hydrogen has been introduced from the surface; hydrogen will preferentially diffuse along grain boundaries. The formation of nickel hydride can be observed along grain boundaries after electrochemical hydrogen charging [25]. At $373 \mathrm{~K}$, hydrogen escapes from nickel vacancies due to its low detrapping energy of $0.91 \mathrm{eV}$ (present work) or $0.98 \mathrm{eV}$ [20- 22]. This means that nano-voids are formed only by short-range migration. The migration is possible even in $99.99 \%$ purity nickel. This result shows that in larger nano-voids some hydrogen atoms will remain. By increasing the annealing temperature, the long lifetime $\tau_{2}$ decreases significantly while its intensity $\mathrm{I}_{2}$ increases between $423 \mathrm{~K}$ and $473 \mathrm{~K}$, which is due to the decomposition of nano-voids and subsequent formation of other types of defects. The positron lifetime value of 169 ps at 473 $\mathrm{K}$ corresponds to positron annihilation in SFT10 [17]. Upon further annealing at $573 \mathrm{~K}$, the average positron lifetimes are between 113 ps and 117 ps, which indicate the presence of some hydrogen-induced defects. These lifetimes correspond to the values observed at edge dislocations and jogs on edge dislocations in nickel [11].

\subsection{Hydrogen charging and annealing of nickel neutron-irradiated to a dose of $1.0 \times 10^{-4} \mathrm{dpa}$}


Hydrogen charging and isochronal annealing have been performed on the nickel specimen neutron-irradiated to a dose of $1.0 \times 10^{-4} \mathrm{dpa}$. The duration of hydrogen charging was 3 $\mathrm{h}$ for the first run and $6 \mathrm{~h}$ for the second run, respectively. The results are shown in Fig. 4.

Fig. 4. Effects of hydrogen charging and isochronal annealing on (a) long lifetime and (b) short lifetime of positrons, and their intensities, in nickel neutron-irradiated at $573 \mathrm{~K}$ for $2.5 \mathrm{~h}$ (irradiation dose: $1.0 \times 10^{-4} \mathrm{dpa}$ ).

The lifetimes, $\tau_{1}$ and $\tau_{2}$, decrease due to hydrogen trapping at vacancies and vacancy clusters. The longer hydrogen charging indicates a larger reduction in the positron lifetimes. This result shows that more hydrogen atoms are trapped in vacancy clusters. If we ignore hydrogeninduced defects, our calculation indicates that nanovoids of 3-5 vacancies created by neutron irradiation absorb at most one hydrogen atom by electrical charging. The intensity of the long lifetime increases, which is mainly attributed to the introduction of defects by hydrogen charging. Upon isochronal annealing, positron lifetimes increase sharply at $323 \mathrm{~K}$, for instance, $\tau_{2}$ increases from 208 ps to 224 ps for the second run, while its intensity decreases significantly. The significant increase in the long lifetime indicates the escape of hydrogen from vacancies. This is confirmed by the value of $\tau_{2}$ at $323 \mathrm{~K}$, which is higher than that before hydrogen charging. It is evident that extra vacancy clusters are introduced by the initial annealing after hydrogen charging, as in the case of unirradiated nickel. Upon further annealing at higher temperatures, the long positron lifetime shows a gradual decrease, reaching a minimum at about $523 \mathrm{~K}$, and then it begins to increase. On the other hand, its intensity increases gradually with annealing temperature, attaining its maximum value also at about $523 \mathrm{~K}$. The presented results indicate that a hydrogen-charged neutron-irradiated nickel specimen contains defects induced by both neutron irradiation and hydrogen charging. The long lifetime, $\tau_{2}$, is the positron lifetime of the two processes. Yoshiie et al. [26] observed that in neutron-irradiated nickel, voids have been formed 
in the matrix, far from the grain boundaries. As mentioned in section 3.2, it is reasonable to assume that nano-voids induced by hydrogen charging are mainly concentrated near the surface and grain boundaries. These clusters are not stable due to their location and the vacancy-clusters are decomposed into vacancies and escape to the surface or to grain boundaries by increasing of the annealing temperature to $573, \mathrm{~K}$. This is as in the case of unirradiated nickel. Nano-voids induced by neutron irradiation remain in the matrix, however, below the irradiation temperature at $573 \mathrm{~K}$. The long positron lifetime $\tau_{2}$ is observed to increase at $573 \mathrm{~K}$, this is attributed to the escape of deeply trapped hydrogen atom from nano-voids formed by neutron irradiation in the matrix.

The trapping of positrons in vacancy clusters competes with their annihilation in the bulk. If only one type of trap is present, the following kinetic equations from a simple trapping model [27-29] describe the annihilation of positrons in the defect-free bulk $\left(n_{\mathrm{b}}\right)$ and at defects $\left(n_{\mathrm{d}}\right)$ :

$$
\begin{aligned}
& \frac{\mathrm{d}}{\mathrm{d} t} n_{\mathrm{b}}(t)=-\lambda_{\mathrm{b}} n_{\mathrm{b}}-\kappa n_{\mathrm{b}}, \\
& \frac{\mathrm{d}}{\mathrm{d} t} n_{\mathrm{d}}(t)=-\lambda_{\mathrm{d}} n_{\mathrm{d}}+\kappa n_{\mathrm{b}},
\end{aligned}
$$

where $\kappa$ is the trapping rate and $\lambda_{\mathrm{b}}$ and $\lambda_{\mathrm{d}}$ are the annihilation rates in the bulk and at defects, respectively. The starting condition at time $t=0$ the number of positrons in the bulk and at defects are $n_{\mathrm{b}}(t=0)=1$ and $n_{\mathrm{d}}(t=0)=0$. The solution of the Esq. 1 and 2 could easily be obtained. The normalized positron lifetime spectrum $n(t)$ presented the summation of two exponential components:

$$
n(t)=I_{1} \exp \left(-t / \tau_{1}\right)+I_{2} \exp \left(-t / \tau_{2}\right)
$$

The lifetimes are written as follows: 


$$
\begin{aligned}
\tau_{1} & =\left(\lambda_{\mathrm{b}}+\kappa\right)^{-1} \\
& =\lambda_{1}^{-1}, \\
\tau_{2} & =\lambda_{\mathrm{d}}^{-1} \\
& =\lambda_{2}^{-1}
\end{aligned}
$$

The relative intensities are

$$
I_{1}=1-I_{2},
$$

$I_{2}=\kappa\left(\lambda_{\mathrm{b}}-\lambda_{\mathrm{d}}+\kappa\right)^{-1}$

The trapping rate is derived from the above equations as

$$
\begin{aligned}
\kappa & =\frac{I_{2}}{I_{1}}\left(\lambda_{\mathrm{b}}-\lambda_{2}\right) \\
& =I_{2}\left(\lambda_{1}-\lambda_{2}\right)
\end{aligned}
$$

The results from trapping rate calculation of positron annihilation in the nano-voids are shown in Fig. 5. It is interesting to see from Fig. 5 that in hydrogen charged nickel the trapping rate of positrons in the nano-voids increases.

Fig. 5. Effects of hydrogen charging and isochronal annealing on the positron trapping rate in nano-voids of nickel neutron-irradiated at $573 \mathrm{~K}$ for $2.5 \mathrm{~h}$ (irradiation dose: $1.0 \times 10^{-4} \mathrm{dpa}$ ).

Although pre-existing vacancy clusters that trap hydrogen decrease the trapping rate, the newly formed vacancies near the surface and grain boundaries act effectively with positrons. The trapping rate decreases quickly as a result of the initial vacuum annealing at $323 \mathrm{~K}$, due to the removal of a number of hydrogen atoms by the formation of vacancies and nano-voids. By increasing annealing temperature, the trapping rate shows a significant increase above $473 \mathrm{~K}$ and reaches a maximum value at about $523 \mathrm{~K}$. This result indicates that trapped hydrogen has been released from nano-voids. The complete desorption of hydrogen from nanovoids by annealing at $573 \mathrm{~K}$ and the recovery of vacancy clusters (SFTs) induced by hydrogen charging result in slightly higher positron trapping rate than that observed before hydrogen charging. The data 
indicate the presence of hydrogen-induced defects after hydrogen charging to neutron irradiated nickel to the dose of $1.0 \times 10^{-4} \mathrm{dpa}$.

\subsection{Hydrogen charging and annealing of nickel neutron-irradiated to a dose of $3.0 \times 10^{-3} \mathrm{dpa}$}

The hydrogen charging and annealing experiments in nickel neutron-irradiated to a dose of $3.0 \times 10^{-3}$ dpa have been performed two times. The results obtained were reproducible. The result of the second run is shown in Figs. 6(a) and (b).

Fig. 6. Effects of hydrogen charging and isochronal annealing on (a) positron lifetimes and (b) their intensities for nickel neutron-irradiated nickel at $573 \mathrm{~K}$ for $75 \mathrm{~h}$ (irradiation dose: $3.0 \times 10^{-3}$ dpa). The first and second points at $300 \mathrm{~K}$ represent hydrogen charging for 6 and $12 \mathrm{~h}$.

The long positron lifetimes, $\tau_{2}$ and $\tau_{3}$, decrease. This is due to hydrogen absorption in neutronirradiated nickel. For example, the longer lifetime decreases from 456 ps to 376 and 334 ps after hydrogen charging for 6 and $12 \mathrm{~h}$, respectively. The present DFT calculations using LDA show that the long positron lifetime of $456 \mathrm{ps}$ in nickel is attributable to positron annihilation in a nano-void containing 33 atoms. The nanovoid site is estimated to have a diameter of $0.9 \mathrm{~nm}$ before relaxation. Nanovoids have not been observed by TEM under this irradiation condition in nickel [17]. The TEM experimental data have been in agreement with the fact, that the smallest nanovoids detectable by TEM are typically $2 \mathrm{~nm}$ in diameter. The number of hydrogen atoms in nano-voids, containing 33 vacancies is estimated to be about 18 and 25 after hydrogen charging at current density of $20 \mathrm{~mA} / \mathrm{cm}^{2}$ for 6 and $12 \mathrm{~h}$, respectively. In Fig. 6(b) is seen that the long lifetime intensities increase upon hydrogen absorption at $300 \mathrm{~K}$ in neutron-irradiated nickel. The increase in intensity $I_{2}$ we explained by the formation of vacancies containing hydrogen. However, the increase in $\mathrm{I}_{3}$ is not attributed to the increase of the nano-void concentration since 
hydrogen absorption is not expected to increase the nanovoid density. This could be caused by the increase in the positron trapping rate in nano-voids due to the strong Coulomb repulsion from ion cores and hydrogen atoms trapped at interstitial sites. As has been already mentioned the migration energy of interstitial hydrogen is $0.41 \mathrm{eV}$ [21], this fact confirms our result that hydrogen easily escapes to the surface and grain boundaries. We should note that interstitial hydrogen atoms would persist even at $300 \mathrm{~K}$ due to existing of trapping sites such as impurities and dislocations in the matrix. Interstitial hydrogen in the matrix of non-irradiated and low dose irradiated nickel has not been detected at the formation of vacancies. In Fig. 6(a) are shown the results from isochronal annealing which was conducted after hydrogen charging. The long positron lifetimes components are seen to increase gradually upon annealing between 373 and $623 \mathrm{~K}$. Our results at first show that the intensities decrease upon annealing at $373 \mathrm{~K}$ and $623 \mathrm{~K}$ $\left(\mathrm{I}_{2}\right)$ or $573 \mathrm{~K}\left(\mathrm{I}_{3}\right)$, as can be seen in Fig. 6(b).

In the present work the positron trapping rate calculations for different types of defects in neutron-irradiated nickel have been performed. Eqs. (1) and (2) are easily expanded by including two types of defects with different annihilation rates, $\lambda_{\mathrm{d} 1}\left(\lambda_{2}\right)$ and $\lambda_{\mathrm{d} 2}\left(\lambda_{3}\right)$. The trapping rates $\kappa_{1}$ and $\kappa_{2}$ are given by the following equations:

$$
\begin{aligned}
& \kappa_{1}=I_{2}\left(\lambda_{1}-\lambda_{2}\right), \\
& \kappa_{2}=I_{3}\left(\lambda_{1}-\lambda_{3}\right)
\end{aligned}
$$

Usually, the trapping rate of different type of defect is assumed to be proportional to its density, $C_{i}$

$$
\kappa_{i}=\mu_{i} C_{i}
$$

where $\mu_{i}$ is the specific trapping rate determined by the defect type. The positron trapping rates $\kappa_{1}$ for SFTs, vacancy, divacancy and $\kappa_{2}$ for nano-voids, created by hydrogen charging and 
neutron irradiated nickel to $3 \times 10^{-3}$ dpa are shown in Fig. 7. The present results show that the trapping rate $\kappa_{1}$ is 3 to 4 times higher than $\kappa_{2}$.

Fig. 7. Effect of hydrogen charging and isochronal annealing on the positron trapping rate in different types of defects in nickel neutron-irradiated at $573 \mathrm{~K}$ for $75 \mathrm{~h}$ (irradiation dose: $3.0 \times 1^{3}$ dpa).

This is can be explained by the higher trapping cross sections of SETs for positrons than nanovoids trapping of positrons or the concentration of SFTs is much higher than the nano-voids. We found that the positron trapping rates increase due to the of hydrogen charging. The decrease at the trapping rate as upon annealing at $373 \mathrm{~K}$ is due to the annihilation of interstitial hydrogen. Experimental data of Tanabe et al. [30] suggest that, in the case of deuterium-ion-implanted nickel, the thermal desorption of deuterium occurred between 503 and $603 \mathrm{~K}$. In their experiment, deuterium was trapped by irradiation-induced defects. In the same experiment the heating rate was very high. That is why for our experiments we could reasonably conclude that the trapped hydrogen atoms in nanovoids of neutron-irradiated nickel undergo complete desorption upon isochronal annealing between 473 and $573 \mathrm{~K}$. Our results show that trapping rates decrease above $523 \mathrm{~K}$, which corresponds to the desorption of hydrogen in nano-voids. Conversely, trapping rates at $573 \mathrm{~K}$ are much higher than those observed before hydrogen charging. This indicates that in a specimen neutron irradiated to $3 \times 10^{-3}$ dpa hydrogen-induced defects exist. These defects are completely annihilated by annealing at $623 \mathrm{~K}$. It is noted that, during isochronal annealing, the long positron lifetime $\tau_{2}$ of the low dose irradiated specimen was greatly influenced by hydrogen charging, while that of the high dose irradiated specimen was not affected by hydrogen charging. We consider that there are two possible explanations for the results. The first one is that the effect of hydrogen charging on the nucleation of defects is 
small in the high dose neutron irradiated nickel, because hydrogen is preferentially trapped by pre-existing nano-voids rather than creating new vacancies. The other is that positron annihilation in the high dose neutron irradiated specimen is predominated by annihilation in neutron irradiation induced nanovoids owing to their high trapping rate of positrons.

\section{Conclusion}

Positron lifetime spectroscopy was used to study the electronic structure effects in electrochemically hydrogen charged and neutron irradiated nickel. In this study for the first time, hydrogen is detected after hydrogen charging in nickel irradiated to different neutron doses. The nano-voids in neutron-irradiated nickel are active positron traps. If they are once bound with hydrogen, they become less effective in the trapping of positrons. The recently developed CDB technique has a possibility to detect the amount of hydrogen in nano-voids. By combining the positron lifetime and $\mathrm{CDB}$ measurements, we may estimate the size of nano-voids and the number of gas atoms in them without distortion and heating of the specimen. The combined techniques used could be applied for estimation of the degradation of candidate nuclear materials for nuclear fusion and fission reactors. The positron lifetimes in neutron-irradiated nickel were shortened by electrically hydrogen charging due to hydrogen trapping in the nano-voids. It was established that the positron-trapping rate in small size nano-voids was 3 to 4 times higher than that in the larger size nano-voids.

\section{Acknowledgement}

The authors are indebted to Dr. S. Booth (UK) for his recommendations and linguistic errors correction of the manuscript. We would like to express our thanks to Dr. A. Hashizume of ESCO Ltd. for measurement of the TDS spectrum. One of the authors (T.T) is indebted to the JSPS. 


\section{References}

1. R. N. West, Positron Studies of Condensed Matter, Advances in Phys. 22 (1973) 263.

2. P. J. Schultz and K. G. Lynn, Rev. Mod. Physics 60, 701 (1988).

3. M. J. Puska, R. M. Nieminen, Rev. Mod. Phys. 66, 841 (1994).

4. K.O. Jensen, R. M. Nieminen, Phys. Rev. B 36, 8219 (1987).

5. K. G. Lynn, J. R. MacDonald, R. A. Boie, L. C. Feldman, J. D. Gabbe, M. F. Robbins, E. Bonderup and J. Golovchenko, Phys. Rev. Lett. 38, 241 (1977).

6. P. A. Kumar, M. Alatalo, V. J. Ghosh, A. C. Kruseman, B. Neilsen and K. G. Lynn, Phys. Rev. Lett. 77, 2097 (1996).

7. S. Linderoth, J. Phys. Cond. Matters 1, SA55 (1989).

8. Y. C. Wu, C. Wu, Y. Itoh and Y. Ito, Physica Status Solidi (b) 193, 307 (1996).

9. T. Troev, E. popov, P. Staikov, N. Nankov and T. Yoshiie, NIM B 267, 535 (2009

10. Q. Xu, T. Ishizaki, K. Sato, T. Yoshiie and S. Nagata, Materials Transactions 47, 2885(2006).

11. H. Ohkubo, S. Sugiyama, K. Fukuzato, M. Takenaka, N. Tsukuda, E. Kuramoto, J. Nucl. Mater. 283-287, 858(2000)

12. T. Yoshiie, Y. Hayashi, S. Yangita, Q. Xu, T. Satoh, H. Tsujimoto, T. Kozuka, K. Kamae, K. Mishima, S. Shiroya, K. Kobayashi, M. Utsuro, Y. Fujita, NIM A 498, 522(2003).

13. P. Kirkegaard, N.J. Peterson, M. Eldrup, PATFIT-88, Risø Natl. Lab. M-2740 (1989).

14. E. Kuramoto, H. Abe, M. Takenaka, F. Hori, Y. Kamimura, M. Kimura, K. Ueno, J. Nucl. Mater.239, 54 (1996).

15. H. Ohkubo, Z. Tang, Y. Nagai, M. Hasegawa, T. Tawara, M. Kiritani, Mater. Sci. Eng. A 350, 95 (2003).

16. E. Kuramoto, T. Tsutsumi, K. Ueno, M. Ohmura, Y.Kamimura, Comp.Mater. Sci. 14, 28 (1999).

17. T. Yoshiie, Q. Xu, Y. Satoh, H. Ohkubo, M. Kiritani, J. Nucl. Mater. 283-287, 229 (2000). 
18. Y. Fukai, N. Okuma, Phys. Rev. Lett. 73, 1640 (1994).

19. Y.Shirai, H. Araki, T. Mori, W. Nakamura, K. Sasaki, J. Alloys Comp. 330-332, 125(2002).

20. T. Ishizaki, Q.Xu, T. Yoshiie, S. Nagata, T. Troev, J. Nucl. Mat. 307-311, 961 (2002).

21. J. Völkl and Alefeld, "Diffusion of hydrogen in metals (basics properties)" in Top. Appl. Phys. Vol.28, Springer-Verlag, 1978, p321.

22. H. Rajainmäki, S. Linderoth, H. E. Hansen, R. M. Nieminen, J. Phys. F 18, 1109 (1988).

23. B. L. Shivachev, T. Troev and T. Yoshiie, J. Nucl. Mater.306, 105(2002).

24. M.Kiritani, M. Konno, T. Yoshiie, S. Kojima, Mater. Sci. Forum 15-18, 181 (1987).

25. A. M. Brass, A. Chanfreau, Acta Mater. 44, 3823(1995).

26. T. Yoshiie, T. Ishizaki, Q. Xu, Y. Satoh, M. Kiritani, J. Nucl. Mater. 307-311, 924 (2002).

27. W. Brandt, in "Positron Annihilation", A.T. Stewart, L. O. Rollig (Eds.), Academic Press, New York, 1967, p. 155.

28. D. C. Connors, R. N. West, Phys. Lett. A 30, 24 (1969).

29. M. Doyama, J. Phys. Soc. Jpn. 33, 1495 (1972).

30. T. Tanabe, H. Hirano, S. Imoto, J. Nucl. Mater. 151, 38 (1987). 
Table 1. Positron annihilation lifetimes and their intensities for well-annealed nickel and neutron-irradiated nickel.

\begin{tabular}{lllllll}
\hline \hline Neutron irradiation at $573 \mathrm{~K}$ & $\tau_{1}$ & $I_{1}$ & $\tau_{2}$ & $I_{2}$ & $\tau_{3}$ & $I_{3}$ \\
Dose $(\mathrm{dpa}) /$ time & $(\mathrm{ps})$ & $(\%)$ & $(\mathrm{ps})$ & $(\%)$ & $(\mathrm{ps})$ & $(\%)$ \\
\hline $0 / 0$ & $110 \pm 1$ & 100 & & & & \\
$6.7 \times 10^{-6} / 10 \mathrm{~min}$ & $77 \pm 2$ & $52 \pm 0.9$ & $187 \pm 4$ & $48 \pm 0.9$ & & \\
$4.0 \times 10^{-5} / 1 \mathrm{~h}$ & $89 \pm 2$ & $79 \pm 1.1$ & $206 \pm 3$ & $21 \pm 0.6$ & & \\
$1.0 \times 10^{-4} / 2.5 \mathrm{~h}$ & $92 \pm 3$ & $62 \pm 1.2$ & $221 \pm 4$ & $37 \pm 0.8$ & & \\
$3.0 \times 10^{-3} / 75 \mathrm{~h}$ & $68 \pm 2$ & $31 \pm 0.5$ & $177 \pm 3$ & $57 \pm 1.1$ & $456 \pm 12$ & $12 \pm 1.3$
\end{tabular}




\section{Figure captions}

Fig. 1. Positron lifetime and its intensity as a function of the electrical hydrogen charging time in nickel.

Fig. 2. Thermal desorption spectrum for electrically hydrogen-charged nickel with a linear temperature ramp of $1 \mathrm{~K} / \mathrm{s}$.

Fig. 3. Effect of isochronal annealing on the positron lifetime and intensity in hydrogen-charged nickel.

Fig. 4. Effects of hydrogen charging and isochronal annealing on (a) long lifetime and (b) short lifetime of positrons, and their intensities, in nickel neutron-irradiated at $573 \mathrm{~K}$ for $2.5 \mathrm{~h}$ (irradiation dose: $1.0 \times 10^{-4} \mathrm{dpa}$ ).

Fig. 5. Effects of hydrogen charging and isochronal annealing on the positron trapping rate in nano-voids of nickel neutron-irradiated at $573 \mathrm{~K}$ for $2.5 \mathrm{~h}$ (irradiation dose: $1.0 \times 10^{-4} \mathrm{dpa}$ ).

Fig. 6. Effects of hydrogen charging and isochronal annealing on (a) positron lifetimes and (b) their intensities for nickel neutron-irradiated nickel at $573 \mathrm{~K}$ for $75 \mathrm{~h}$ (irradiation dose: $3.0 \times 10^{-3}$ dpa). The first and second points at $300 \mathrm{~K}$ represent hydrogen charging for 6 and $12 \mathrm{~h}$.

Fig. 7. Effect of hydrogen charging and isochronal annealing on the positron trapping rate in different types of defects in nickel neutron-irradiated at $573 \mathrm{~K}$ for $75 \mathrm{~h}$ (irradiation dose: $3.0 \times 1^{3}$ dpa). 


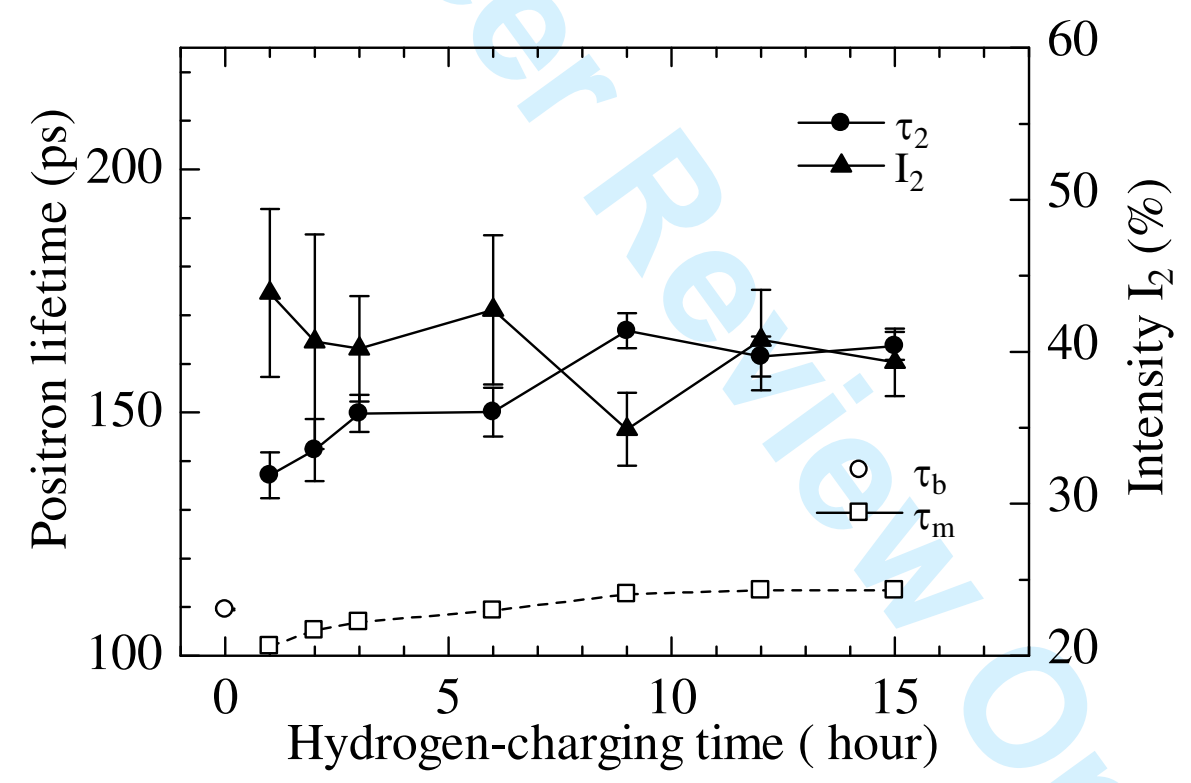

Fig. 1. Effect of electrical hydrogen charging on positron lifetime and intensity in nickel. 


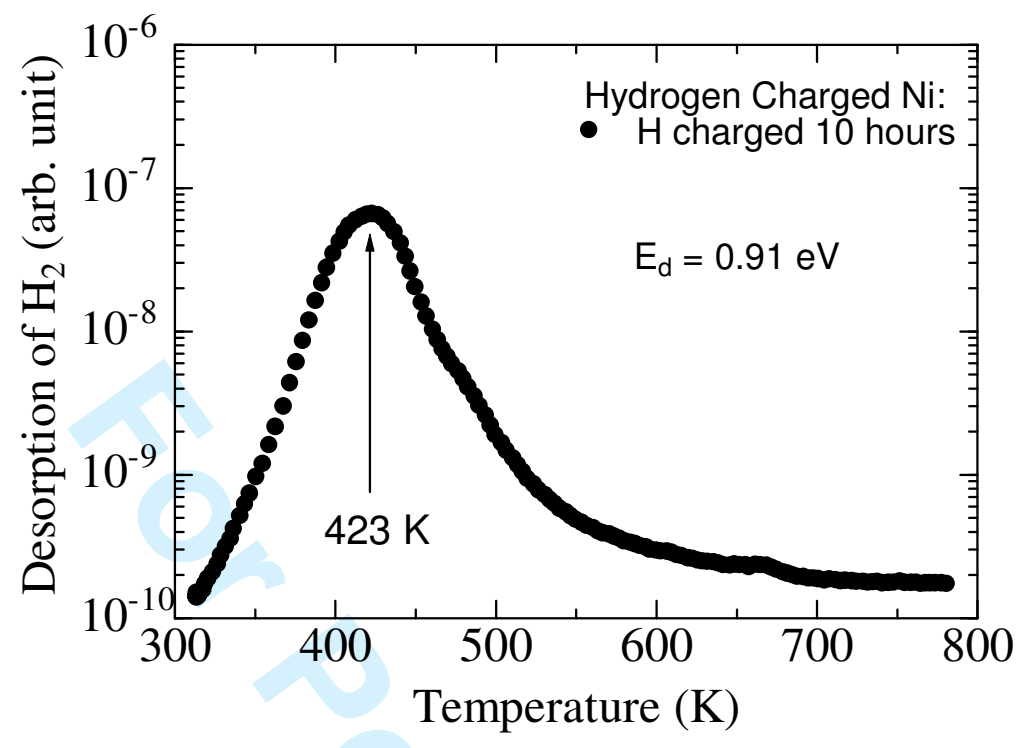

Fig. 2. Thermal desorption spectrum for electrically hydrogen-charged nickel with a linear temperature ramp of $1 \mathrm{~K} / \mathrm{s}$.

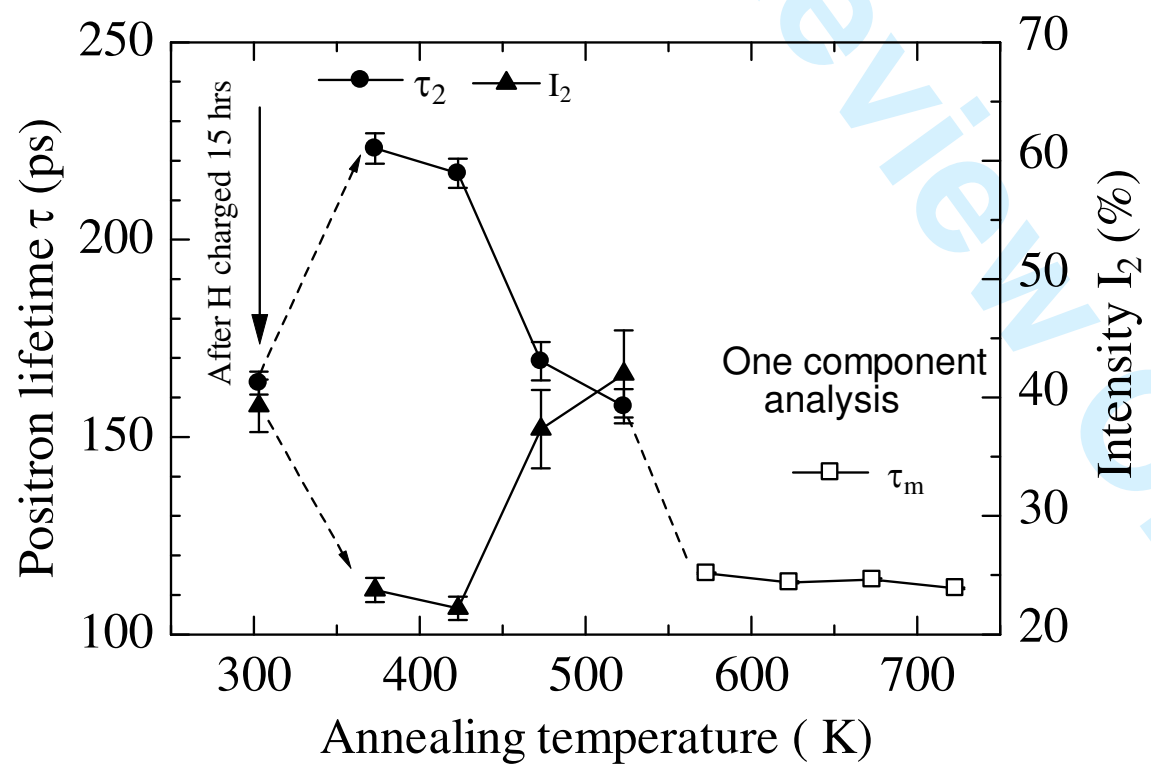

Fig. 3. Effect of isochronal annealing on the positron lifetime and intensity in hydrogen-charged nickel. 

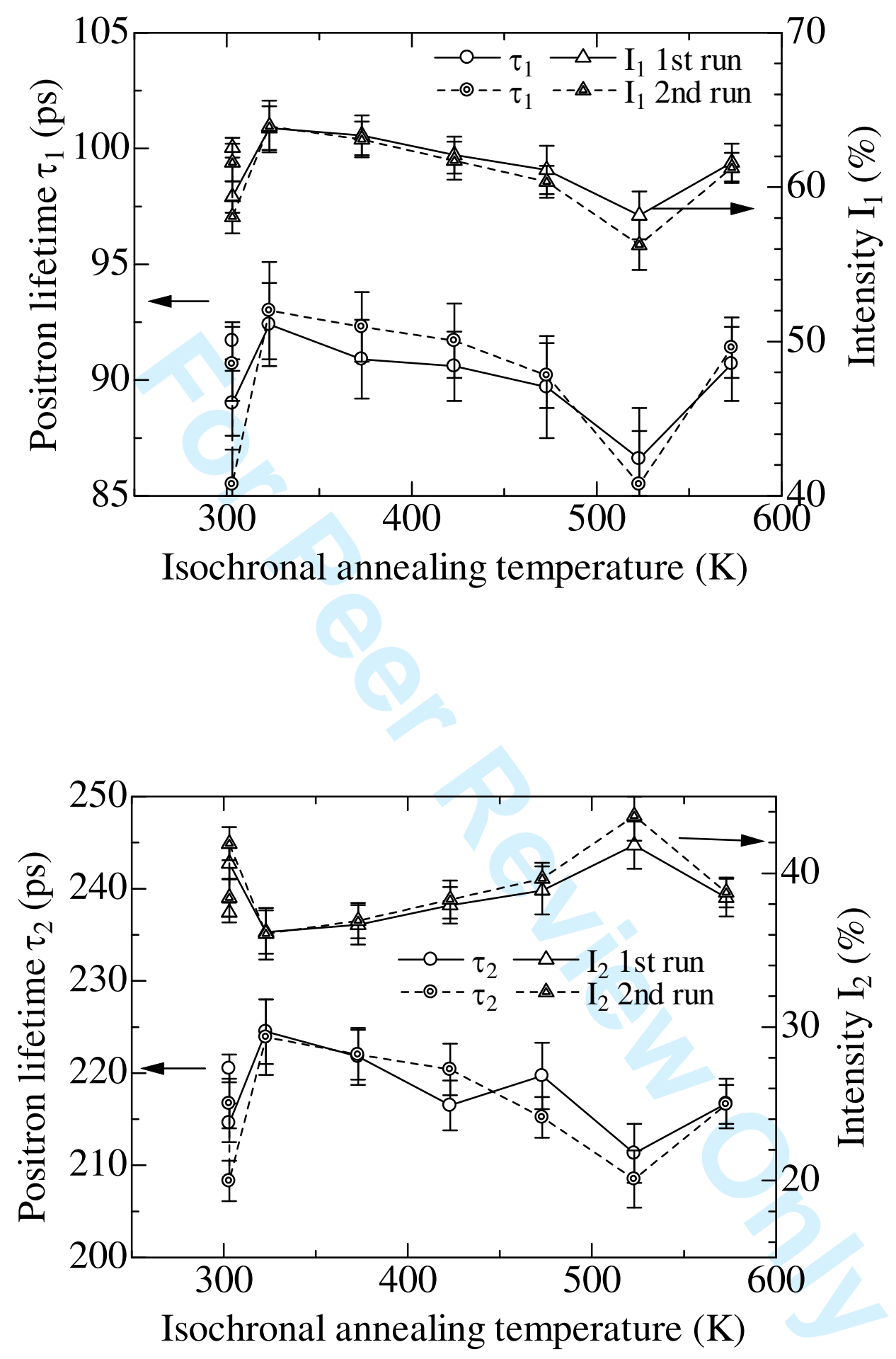

Fig. 4. Effects of hydrogen charging and isochronal annealing on (a) long lifetime and (b) short lifetime of positrons, and their intensities, in nickel neutron-irradiated at $573 \mathrm{~K}$ for $2.5 \mathrm{~h}$ (irradiation dose: $1.0 \times 10^{-4} \mathrm{dpa}$ ). 


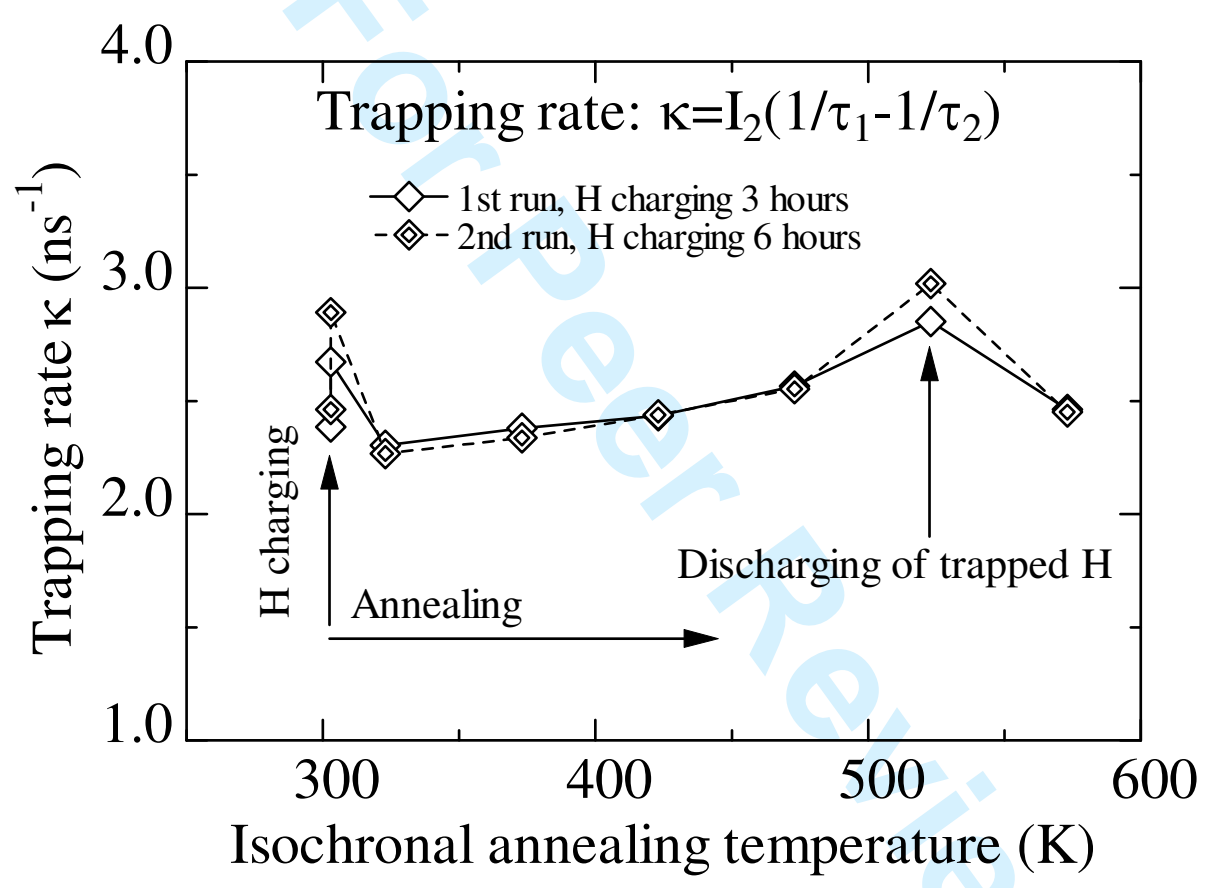

Fig. 5. Effects of hydrogen charging and isochronal annealing on the positron trapping rate in nano-voids of nickel neutron-irradiated at $573 \mathrm{~K}$ for $2.5 \mathrm{~h}$ (irradiation dose: $1.0 \times 10^{-4} \mathrm{dpa}$ ). 

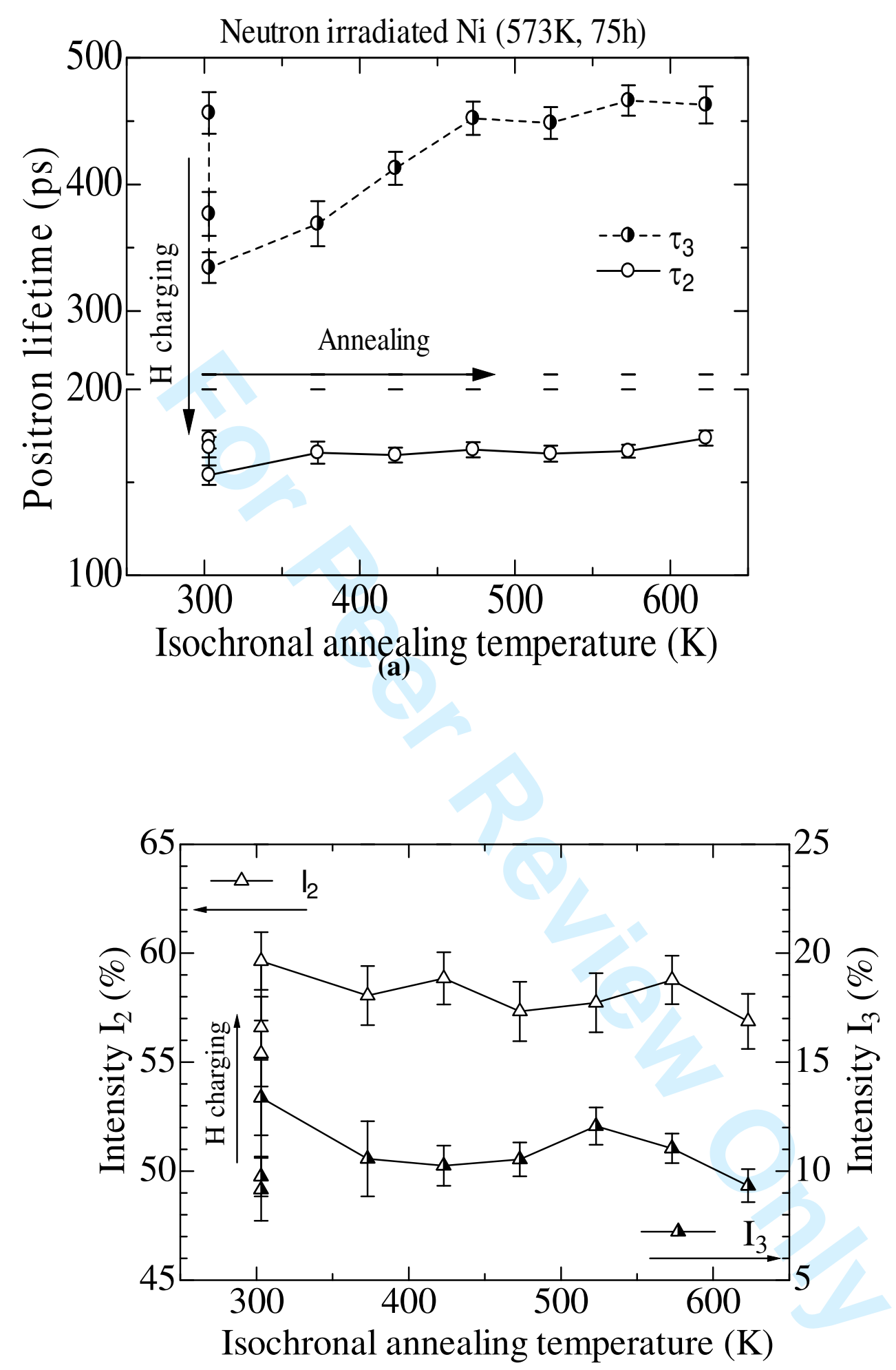

(b)

Fig. 6. Effects of hydrogen charging and isochronal annealing on (a) positron lifetimes and (b) their intensities for nickel neutron-irradiated nickel at $573 \mathrm{~K}$ for $75 \mathrm{~h}$ (irradiation dose: $3.0 \times 10^{-3}$ dpa). The first and second points at $300 \mathrm{~K}$ represent hydrogen charging for 6 and $12 \mathrm{~h}$. 


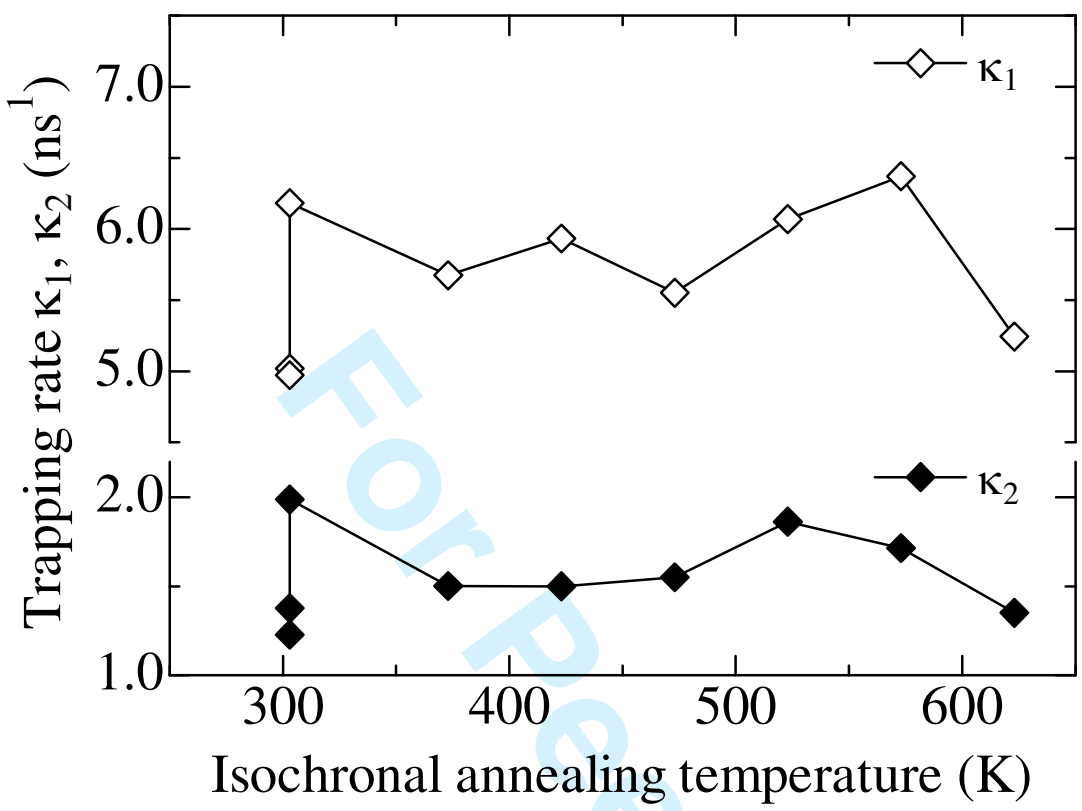

Fig. 7. Effect of hydrogen charging and isochronal annealing on the positron trapping rate in different types of defects in nickel neutron-irradiated at $573 \mathrm{~K}$ for $75 \mathrm{~h}$ (irradiation dose: $3.0 \times 10^{-}$ 3 dpa). 
Table 1. Positron annihilation lifetimes and their intensities for well-annealed nickel and neutron-irradiated nickel.

\begin{tabular}{lllllll}
\hline \hline Neutron irradiation at $573 \mathrm{~K}$ & $\tau_{1}$ & $I_{1}$ & $\tau_{2}$ & $I_{2}$ & $\tau_{3}$ & $I_{3}$ \\
Dose $(\mathrm{dpa}) /$ time & $(\mathrm{ps})$ & $(\%)$ & $(\mathrm{ps})$ & $(\%)$ & $(\mathrm{ps})$ & $(\%)$ \\
\hline $0 / 0$ & $110 \pm 1$ & 100 & & & \\
$6.7 \times 10^{-6} / 10 \mathrm{~min}$ & $77 \pm 2$ & $52 \pm 0.9$ & $187 \pm 4$ & $48 \pm 0.9$ & & \\
$4.0 \times 10^{-5} / 1 \mathrm{~h}$ & $89 \pm 2$ & $79 \pm 1.1$ & $206 \pm 3$ & $21 \pm 0.6$ & & \\
$1.0 \times 10^{-4} / 2.5 \mathrm{~h}$ & $92 \pm 3$ & $62 \pm 1.2$ & $221 \pm 4$ & $37 \pm 0.8$ & & \\
$3.0 \times 10^{-3} / 75 \mathrm{~h}$ & $68 \pm 2$ & $31 \pm 0.5$ & $177 \pm 3$ & $57 \pm 1.1$ & $456 \pm 12$ & $12 \pm 1.3$ \\
\hline \hline
\end{tabular}




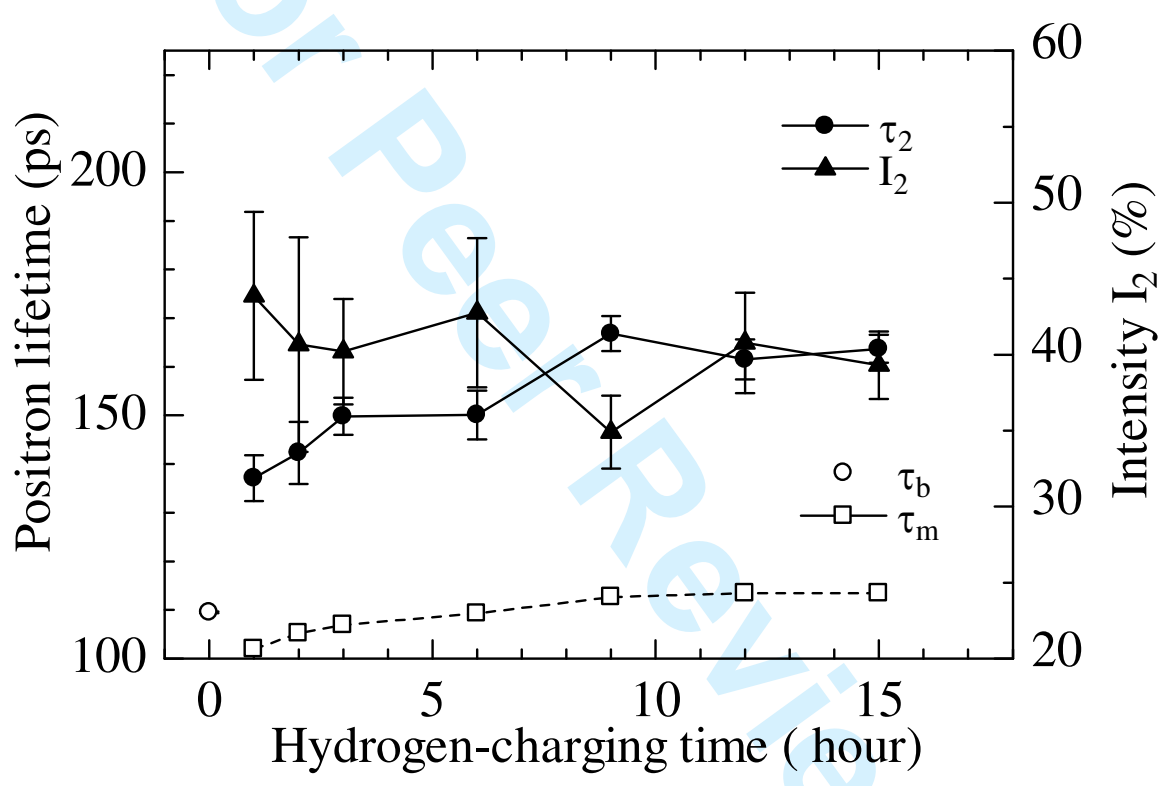




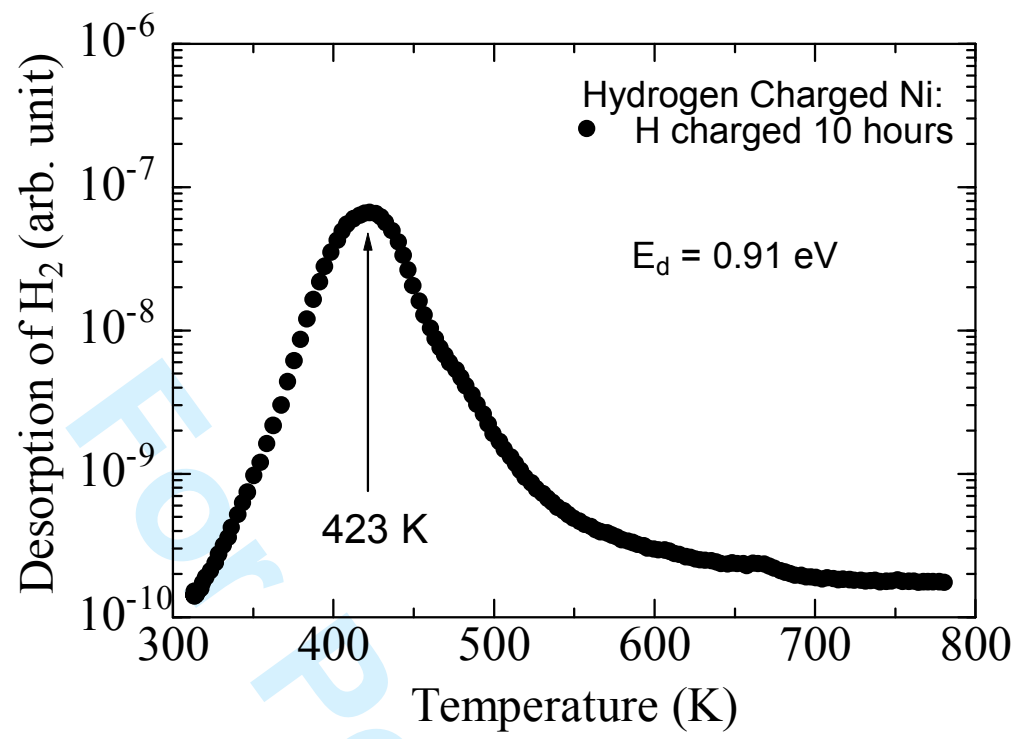




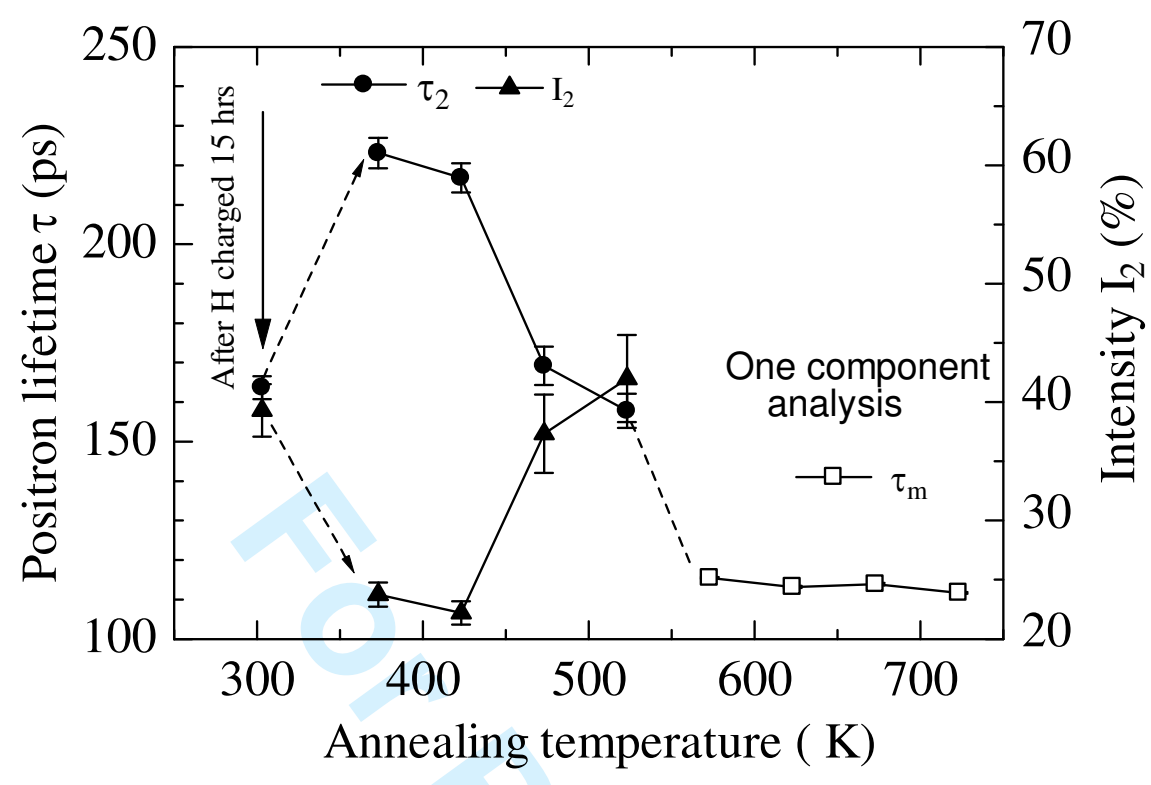



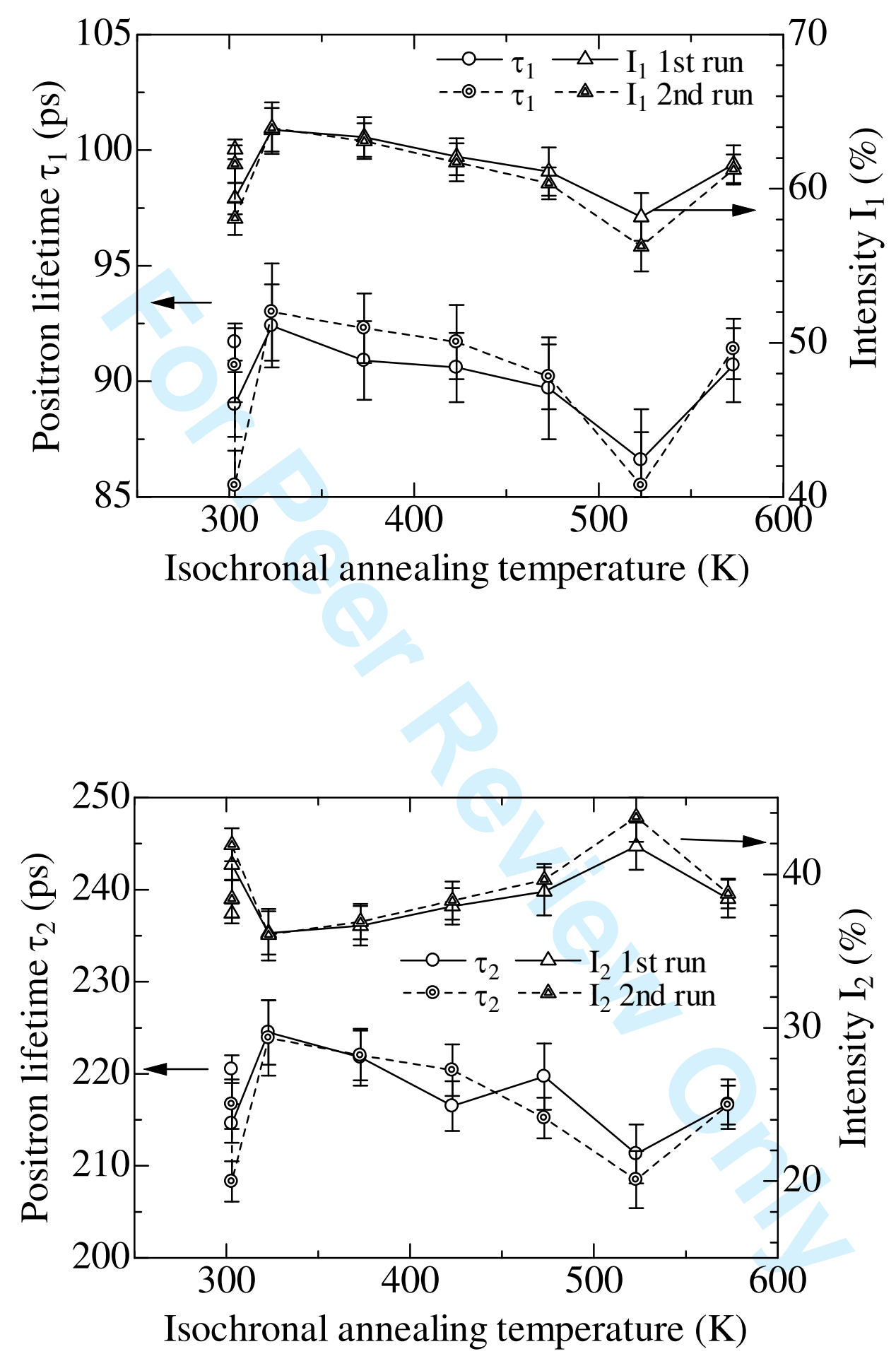


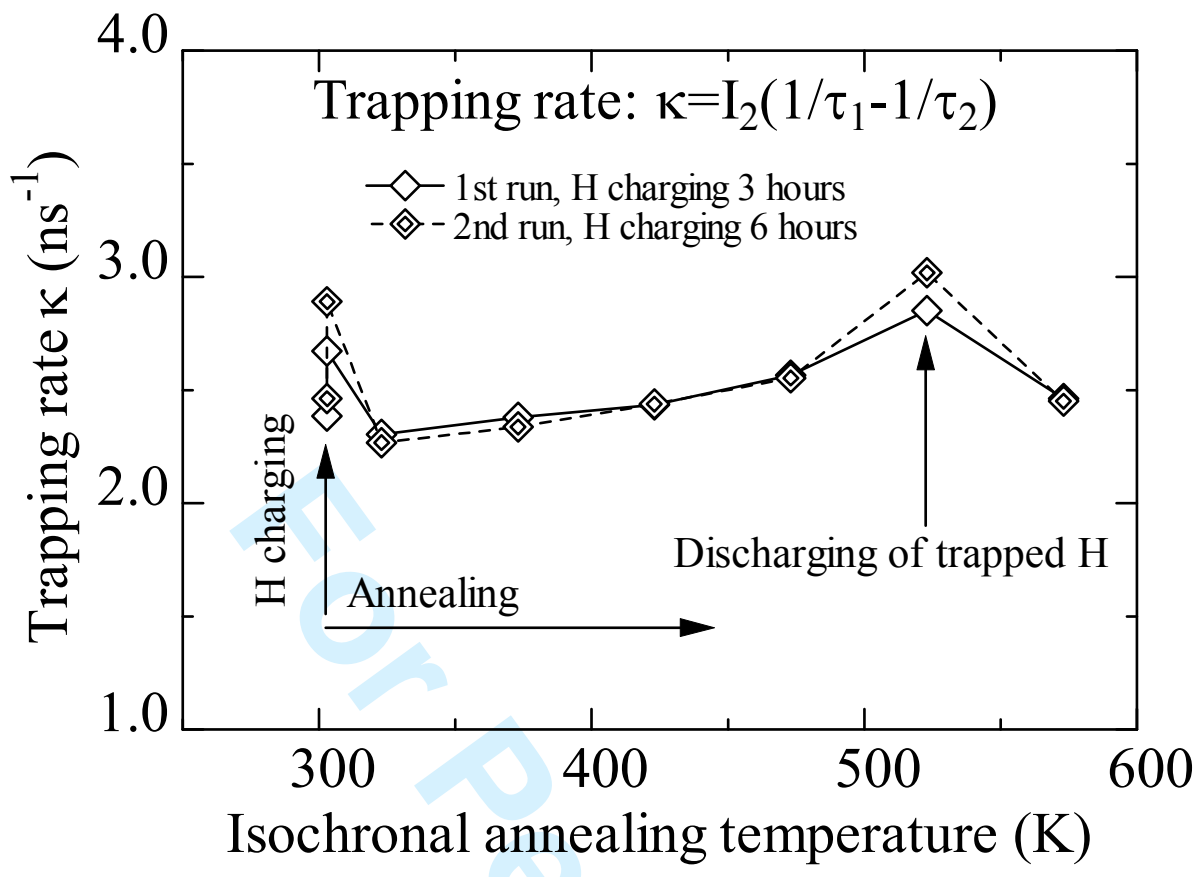



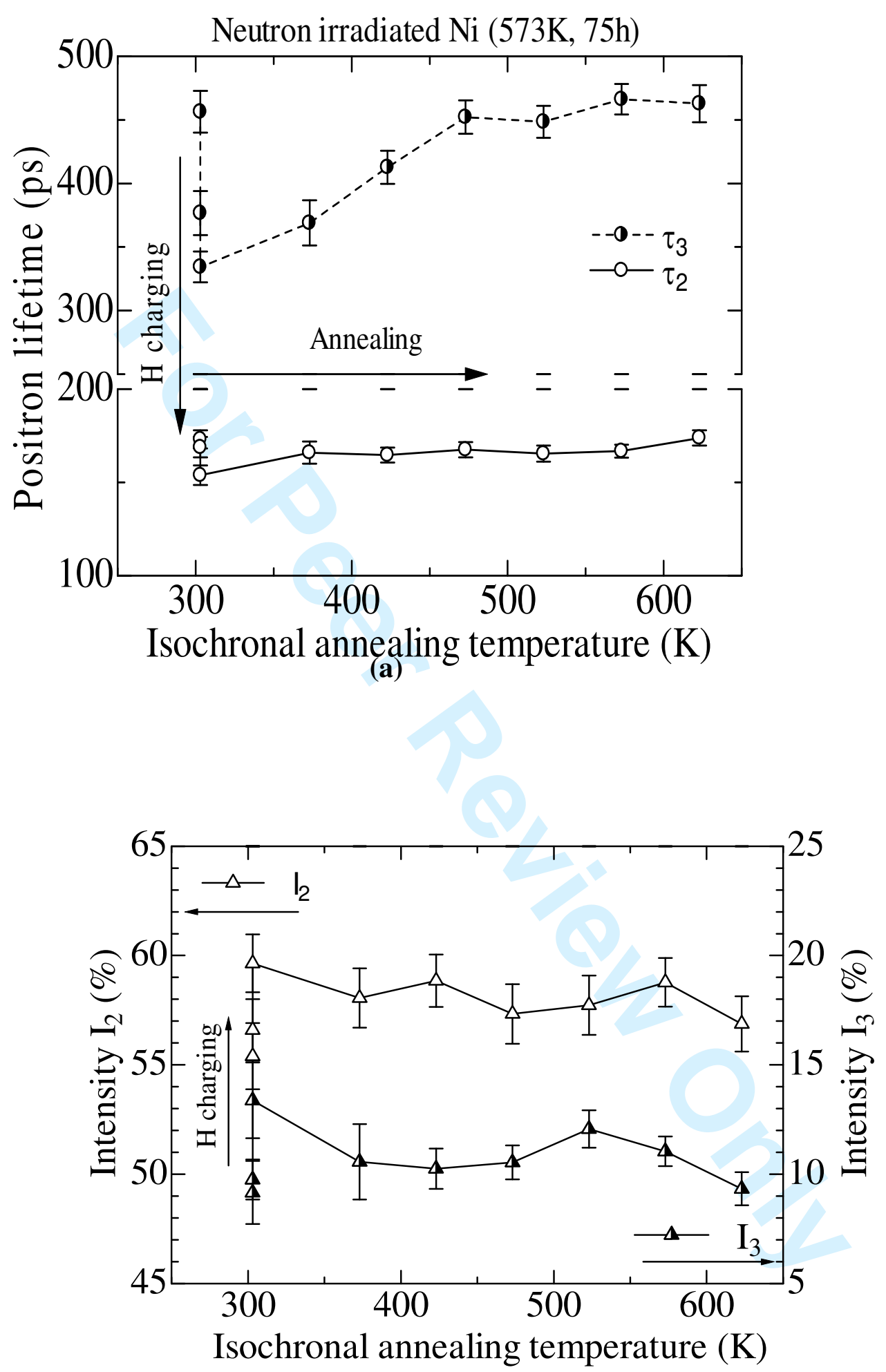

(b) 


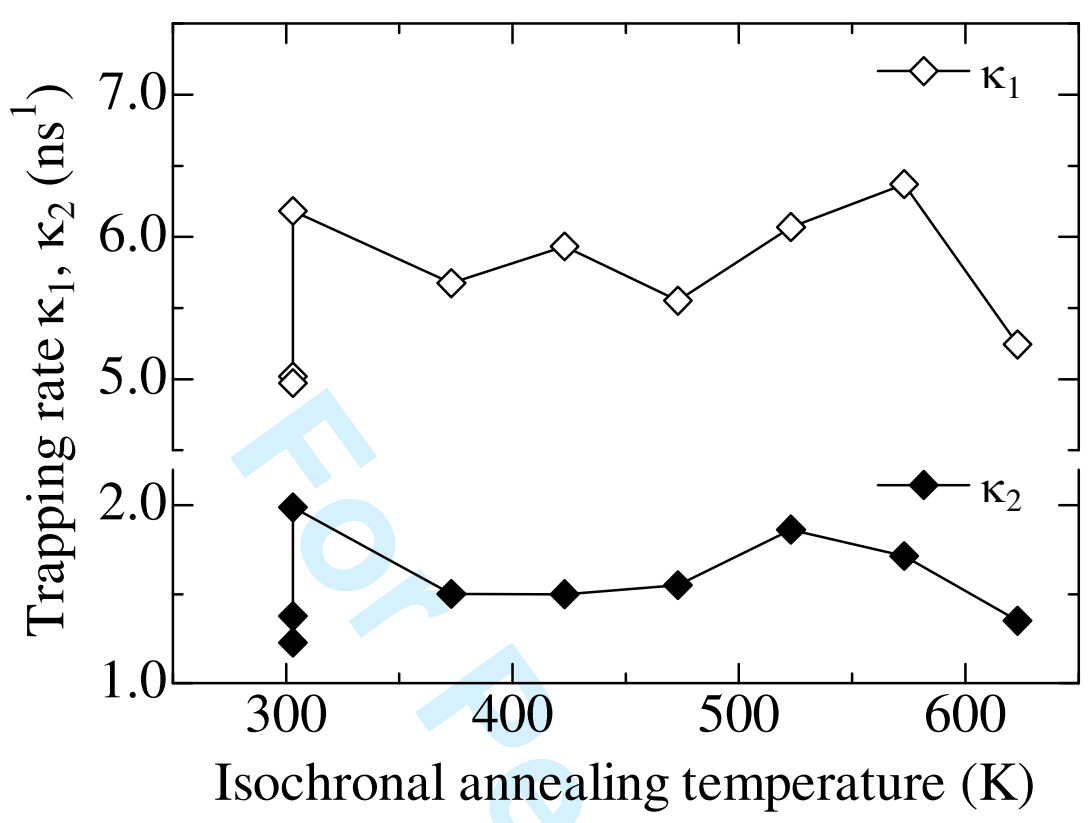

